\title{
Clinical efficacy of dalbavancin for the treatment of acute bacterial skin and skin structure infections (ABSSSI)
}

This article was published in the following Dove Press journal:

Therapeutics and Clinical Risk Management

7 June 2016

Number of times this article has been viewed

\author{
Kimberly D Leuthner' \\ Kristin A Buechler' \\ David Kogan' \\ Agafe Saguros' \\ H Stephen Lee ${ }^{2}$ \\ 'Department of Pharmaceutical \\ Services, University Medical Center \\ of Southern Nevada, Las Vegas, NV, \\ USA; ${ }^{2}$ Roseman University of Health \\ Sciences College of Pharmacy, \\ Henderson, NV, USA
}

\begin{abstract}
Acute bacterial skin and skin structure infections (ABSSSI) are a common disease causing patients to seek treatment through the health care system. With the continued increase of drug-resistant bacterial pathogens, these infections are becoming more difficult to successfully cure. Lipoglycopeptides have unique properties that allow the drug to remain active toward both common and challenging pathogens at the infected site for lengthy periods of time. Dalbavancin, a new lipoglycopeptide, provides two unique dosing regimens for the treatment of ABSSSI. The original regimen of $1,000 \mathrm{mg}$ intravenous infusion followed by a $500 \mathrm{mg}$ intravenous infusion after a week has been shown as safe and effective in multiple, randomized noninferiority trials. These studies also demonstrated that dalbavancin was similar to standard regimens in terms of both safety and tolerability. Recently a single $1,500 \mathrm{mg}$ dose was demonstrated to be equivalent to the dalbavancin two-dose regimen for treating ABSSSI. With the introduction of dalbavancin, clinicians have the option to provide an intravenous antimicrobial agent shown to be as effective as traditional therapies, without requiring admission into the hospitals or prescribing a medication which may not be utilized optimally. Further understanding of dalbavancin and its unusual properties can provide unique treatment situations with potential benefits for both the patient and the overall health care system, which should be further explored.
\end{abstract}

Keywords: dalbavancin, lipoglycopeptide, ABSSSI, skin and skin structure infection, dosing schedule, Gram-positive resistance

\section{Introduction}

Acute bacterial skin and skin structure infections (ABSSSI) are a common cause for individuals to seek medical care. ABSSSI are infections of the skin and surrounding tissues; common clinical presentations of ABSSSI include skin lesions with redness, induration, and edema, as well as report of pain and significant discomfort by the affected individuals. ${ }^{1}$ In the recent past, the United States Food and Drug Administration (FDA) has defined ABSSSI as bacterial infection of the skin with a minimum lesion area of at least $75 \mathrm{~cm}^{2}$, and with encompassing diseases such as cellulitis, erysipelas, wound infections, and major cutaneous abscesses. ${ }^{2}$ Unlike many other types of infections, patients' contacts to health care environments and immune status are not predisposing factors for developing ABSSSI. In addition to traditional etiologic agents such as Staphylococcus spp. and Streptococcus spp., clinically challenging organisms such as methicillin-resistant Staphylococcus aureus (MRSA) are becoming leading causes of ABSSSI throughout the United States. This has resulted in increased treatment failure with conventional drug therapy, and ultimately higher health care resource utilization and overall costs. This trend was clearly established by Pallin et $\mathrm{al}^{3}$ who demonstrated almost three times
Correspondence: Kimberly D Leuthner University Medical Center of Southern Nevada, I 800 W Charleston Blvd., Las Vegas, NV 89102, USA

Tel +l 7023831878

Fax +l 7023832052

Email kimberly.leuthner@umcsn.com
Therapeutics and Clinical Risk Management 2016:12 93 |-940

931

Dovepress

http://dx.doi.org/1 0.2147/TCRM.\$86330 (c) (i) (5) 2016 Leuthner et al. This work is published and licensed by Dove Medical Press Limited. The full terms of this license are available at https://www.dovepress.com/terms.php cc. ${ }_{\mathrm{BY}} \mathrm{NC}$ and incorporate the Creative Commons Attribution - Non Commercial (unported, v3.0) License (http://(creativecommons.org/licenses/by-n/ 3.00 ). By accessing the work you hereby accept the Terms. Non-commercial uses of the work are permitted without any further permission from Dove Medical Press Limited, provided the work is properly attributed. For permission for commercial use of this work, please see paragraphs 4.2 and 5 of our Terms (https://www.dovepress.com/terms.php). 
more patients presenting to the emergency department in 2005 for infections of the skin and soft tissue as compared to 1993. Even with this significant increase shown, the actual infection incidence may be underestimated, as demonstrated by Wilder et al, ${ }^{4}$ who reported less than $50 \%$ of patients who suspect they have a skin infection actually seek medical treatment. Currently, multiple effective treatment options for ABSSSI are available for infections caused by the traditional pathogens. The recently updated treatment guidelines from the Infectious Disease Society of America provided therapeutic recommendations for 46 purulent and nonpurulent ABSSSI that are primarily based on the $\beta$-lactam class of antibiotics. However, when MRSA is a suspected pathogen in ABSSSI, trimethoprim/sulfamethoxazole (TMP/SMX) and doxycycline are suggested as empiric therapy for purulent cases of moderate severity, while other anti-MRSA agents, such as vancomycin, linezolid, or daptomycin, are recommended for treating severe purulent cellulitis. Regardless of antimicrobial therapy, adequate source control for infection, such as draining of abscesses and debridement of nonviable tissues, is necessary. ${ }^{1}$ Duong et $\mathrm{al}^{5}$ evaluated the use of systemic antibiotics after appropriate incision and drainage for abscesses in pediatric patients. Similar treatment success rates were detected in patients receiving TMP/SMX and placebo, and fewer than $6 \%$ of subjects failed therapy in both groups on day 10 after receiving appropriate surgical intervention.

Vancomycin, given intravenously, has traditionally been the mainstay of drug therapy against MRSA, but effective treatment options avoiding hospital admission are clinically attractive. Nevertheless, there are general concerns with the ability to utilize vancomycin appropriately in outpatient therapy. There are also concerns about ever-increasing drug resistance by common pathogens of ABSSSI, such as MRSA, that results in vancomycin becoming increasingly less effective. ${ }^{6}$ Any novel therapy for ABSSSI that is safe and effective against drug-resistant organisms may be a promising therapeutic option and warrants careful evaluation.

Dalbavancin is a semisynthetic lipoglycopeptide antibiotic agent, approved by the FDA for the treatment of ABSSSI therapy in adult patients in May 2014. It was developed to be an improved alternative to the currently available glycopeptides teicoplanin and vancomycin, which are common intravenous agents for ABSSSI associated with MRSA. With a favorable 69 spectrum of antibacterial action and adverse effect profile, as well as a significantly longer drug half-life, dalbavancin offers a promising new alternative in the armory of agents available for treatment of ABSSSI due to Gram-positive pathogens including MRSA., ${ }^{7,8}$ The longer drug half-life allows for a once-weekly dosing interval, and offers an advantage over glycopeptides such as vancomycin for use in the outpatient setting. ${ }^{5}$ This paper will review the basic pharmacokinetic and pharmacodynamics properties of dalbavancin, including the clinical evidence for the utilization of dalbavancin for ABSSSI, and evaluate its potential place in therapy.

\section{Pharmacology and mechanism of action}

Dalbavancin is a synthetic lipoglycopeptide developed from a compound called A40926, which was first isolated from Nonomuraea species. This organism is part of the Actinomycetes family, which has produced many antimicrobial compounds. ${ }^{9}$ The mechanism of activity is as a result of the inhibition of bacterial cell wall synthesis by targeting the terminal D-alanyl-D-alanine residues of the cell wall of susceptible bacteria. Similar to the glycopeptide precursors vancomycin and teicoplanin, this activity results in the disruption of transpeptidase and transglycosylase activities and eventually the inhibition of the cellular wall formation. ${ }^{9-11}$ A40926 maintains the required glycopeptides backbone structure similar to teicoplanin, but with structural changes aiming to provide the ability for drug dimerization and to anchor the lipophilic side chain of dalbavancin in the bacterial membrane such that there is improved interaction and binding affinity of dalbavancin toward its target (Figure 1). This in turn produces more rapid and potent bactericidal activity than with vancomycin, most notably among the staphylococci species. ${ }^{9-12}$

\section{Pharmacokinetics and metabolism Absorption}

Relative to most antibacterial agents, dalbavancin is a large chemical molecule, and it does not undergo absorption when given through the enteral route. As such, intravenous administration is the only way to achieve adequate serum concentrations to treat systemic infections. Summarized in Table 1 are the pharmacokinetic parameters after a single $1,000 \mathrm{mg}$ dose is given. When larger doses are given, pharmacokinetic studies demonstrated that the values of area under the curve over 24 hours $\left(\mathrm{AUC}_{0-24}\right)$ and maximum concentration $\left(C_{\max }\right)$ increased in direct proportion to those of the $1,000 \mathrm{mg}$ dose, suggesting the drug demonstrates linear pharmacokinetic properties. Drug accrual over longer periods was evaluated in healthy volunteers, and no significant drug accumulation occurred over an 8-week period when $500 \mathrm{mg}$ doses of dalbavancin were infused weekly. ${ }^{8,13}$ 


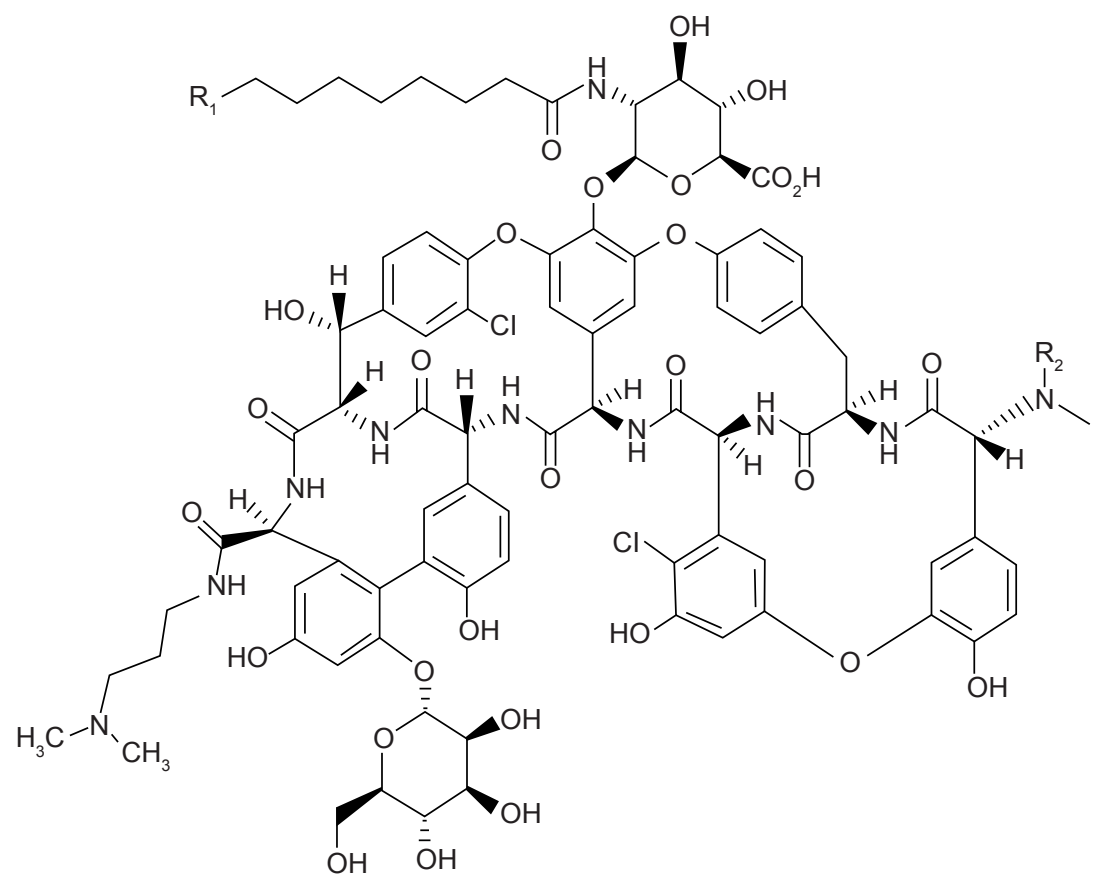

Figure I The chemical structure of dalbavancin.

\section{Distribution}

Dalbavancin demonstrated a high protein binding property, largely binding reversibly to human albumin and other plasma proteins to a much lesser extent. Significant changes in serum drug concentrations and protein binding due to renal or hepatic impairment have not been identified in clinical studies. ${ }^{8,13,14}$ Buckwalter and Dowell ${ }^{14}$ determined that the population kinetics of dalbavancin is best described using a two-compartment model. Within the initial 2 days postinfusion, the plasma (central compartment) concentrations rapidly decrease as the drug distributes into the tissue (periphery compartment). This activity results in a volume of distribution in the peripheral compartment $(11.8 \mathrm{~L})$ being almost three times as large as the central

Table I Pharmacokinetic parameters in healthy subjects

\begin{tabular}{ll}
\hline Parameter & Single I,000 mg dose \\
\hline$C_{\text {max }}(\mathrm{mg} / \mathrm{L})$ & $287(13.9)^{\mathrm{a}}$ \\
$\mathrm{AUC}_{0-24}(\mathrm{mg} \cdot \mathrm{h} / \mathrm{L})$ & $3,185(12.8)^{\mathrm{a}}$ \\
$\mathrm{AUC}_{0-\text { day }}(\mathrm{mg} \cdot \mathrm{h} / \mathrm{L})$ & $\mathrm{II}, 160(4 \mathrm{I} . \mathrm{I})^{\mathrm{b}}$ \\
$\mathrm{AUC}_{0-\infty}(\mathrm{mg} \cdot \mathrm{h} / \mathrm{L})$ & $23,443(40.9)^{\mathrm{b}}$ \\
Terminal $_{1 / 2}(\mathrm{~h})$ & $346(16.5)^{\mathrm{b}, \mathrm{c}}$ \\
$\mathrm{CL}(\mathrm{L} / \mathrm{h})$ & $0.0513(46.8)^{\mathrm{b}}$ \\
\hline
\end{tabular}

Notes: All values are presented as mean (\% coefficient of variation). ${ }^{2}$ Data from 50 healthy subjects. 'Data from 12 healthy subjects. 'On the basis of population pharmacokinetic analyses of patient data, the effective half-life $\left(t_{1 / 2} \beta\right)$ is approximately 8.5 days (204 hours). Data from Dalvance ${ }^{\mathrm{TM}}$ [package insert]. Chicago, IL: Durata Therapeutics; $2014 .{ }^{8}$

Abbreviations: AUC, area under the curve; $C_{m a x}$, maximum concentration: $\mathrm{CL}$, clearance; $t_{1 / 2}$, half-life. compartment $(4.0 \mathrm{~L})$, as well as an overall volume of distribution of $15.7 \mathrm{~L}$ and prolonged terminal half-life. Other studies support these parameters by demonstrating an initial loading dose of $1,000 \mathrm{mg}$ of dalbavancin resulted in a day 8 mean plasma concentration of $30.4 \mathrm{mg} / \mathrm{dL} .{ }^{15}$ Additionally, animal models suggest the liver and kidney have the highest detectible levels of dalbavancin 24 hours after the initial dose administration. However, it appears to distribute relatively equally throughout many tissues, with drug levels detectible in brown fat, kidneys, liver, and skeletal muscle. ${ }^{12,16}$ The drug is also detected in human skin tissues up to 14 days and with drug concentration far above the $\mathrm{MIC}_{90}$ (minimum inhibitory concentration at which $90 \%$ of the isolates are inhibited) of MRSA and $\beta$-hemolytic streptococci. ${ }^{17}$

\section{Metabolism}

When evaluated by in vitro studies, the metabolism of dalbavancin was minimally impacted by the human hepatic CYP450 system. ${ }^{14}$ Further investigations with either inducers or inhibitors of this enzyme system demonstrated no changes in the elimination or clearance of dalbavancin, and the metabolism of model compounds of these CYP systems was not altered by the dalbavancin. Hydroxy-dalbavancin, a minor metabolite that has only been identified in urine, was also not changed in its formation or elimination with these enzyme models. ${ }^{8}, 14$ 


\section{Elimination}

Similar to other predecessor compounds in the glycopeptides class of antibacterial agents, dalbavancin is primarily eliminated through the renal route, with nonrenal routes playing a minor role. Through the urinary tract, $\sim 40 \%$ of the drug is cleared as unchanged molecule, and an additional $12 \%$ removed as hydroxy-dalbavancin. Animal studies suggested $\sim 50 \%$ of unchanged drug could be excreted into the bile; however during initial human trials, only an additional $20 \%$ was identified in feces after a 70 -day postadministration period. ${ }^{12}$ Following the dosing regimen of $1,000 \mathrm{mg}$ on day 1 with a subsequent follow-up dose on day 8 , the serum half-life was determined to be $\sim 346 \pm 16.5$ hours. As with many new medications, limited information is presently available for patients with renal insufficiency. A study with 28 patients determined that the mean plasma concentrations increased by $11 \%$ in patients with mild renal impairment, $35 \%$ in patients with moderate renal impairment, and $47 \%$ in patients with severe renal impairment. Further evaluation in a larger population will be necessary to elucidate exactly how these changes will impact on patient outcomes. On the basis of this limited information, preliminary guidance for dosage adjustment has been suggested. At present, if patients are undergoing hemodialysis, dose adjustments of dalbavancin are not warranted; for patients with an estimated creatinine clearance below $30 \mathrm{~mL} / \mathrm{min}$, the dose should be reduced $25 \%{ }^{8,13}$

\section{Pharmacodynamics}

On the basis of population studies, dalbavancin MICs suggest potency over vancomycin against both Staphylococcus and Enterococcus spp., with $\mathrm{MIC}_{90}$ around 0.06 and $0.12 \mu \mathrm{g} / \mathrm{mL}$, respectively. ${ }^{18}$ In vitro modeling was performed by Bowker et $\mathrm{al}^{19}$ in an attempt to describe the activity of dalbavancin against Gram-positive bacteria. Utilizing various strains of methicillin-susceptible $S$. aureus, MRSA, and vancomycin-intermediate $S$. aureus, the effects of dalbavancin at multiple concentrations were examined. From these simulations, area under the concentration-versus-time curve to MIC ratio (AUC/MIC), rather than a concentration-based relationship, predicted the largest organism response and likely the best description of the mode of killing. This type of activity was further confirmed using in vivo neutropenic lung and thigh animal models in the laboratory of Andes and Craig. ${ }^{20}$ Utilizing many strains of Streptococcus pneumoniae and $S$. aureus including MRSA, multiple simulations were undertaken to evaluate different concentrations and dosing strategies. Results from these studies were in agreement with the in vitro assessment, demonstrating that AUC/MIC activity was strongly correlated with dalbavancin activity.

\section{Spectrum of activity}

The antibacterial activity of dalbavancin is primarily against Gram-positive pathogens, as predicted by the similarities of the chemical structure of dalbavancin to available glycopeptides. Dalbavancin is currently only FDA approved for treating infections caused by susceptible strains of $S$. aureus including MRSA, Streptococcus pyogenes, Streptococcus agalactiae, and the Streptococcus anginosus group. Dalbavancin has increased potency, as reflected by low MICs and susceptibility breakpoints at $<0.12 \mu \mathrm{g} / \mathrm{mL}$ against Streptococcus and Staphylococcus spp. ${ }^{8}$ Multiple in vitro evaluations have shown that dalbavancin is active against many other Gram-positive aerobes such as Enterococcus spp. and Listeria spp., and anaerobic pathogens such as Corynebacterium and Clostridium spp. ${ }^{18,21-23}$ Since the clinical application of dalbavancin has not been established against these organisms not listed in the FDA approval, susceptibility breakpoints have not been established, but the susceptibilities based on in vitro testing appear similar to those listed in the dalbavancin prescribing information. As summarized by Leuthner et al, ${ }^{24}$ the MIC range for many Gram-positive organisms including Streptococcus pneumoniae, and Viridans group Streptococcus spp. are between $<0.03$ and $0.25 \mu \mathrm{g} / \mathrm{mL}$, suggesting that dalbavancin might be successful at treating infections caused by these pathogens. The effects of combinations of drugs are an important aspect to understand for any new agent introduced into use against active infections. Johnson et $\mathrm{al}^{25}$ simulated the use of dalbavancin with nine other antimicrobial agents against a variety of Gram-positive isolates, including some not FDA-approved such as Enterococcus spp. These simulations identified synergy or partial synergy with $90 \%$ of the isolates when dalbavancin was used in combination with oxacillin for MRSA. The majority of the interactions with other compounds and isolates were categorized as either partial synergy (34.4\%), indifference $(33.3 \%)$, or additive activity (22.2\%). Importantly, none of the simulations demonstrated that dalbavancin experienced antagonism in any combination. ${ }^{25}$ Resistance development during treatment and with cumulative use of antibiotics over time is also of significant concern for newly approved compounds. Bacterial isolates were investigated in in vitro evaluations in an attempt to predict future resistance developments. Two independent laboratories undertook very similar simulations of exposing drug-susceptible organisms to various concentrations of dalbavancin below the isolate MIC, using serial passage 
technique to determine the risk for spontaneous mutations. ${ }^{26,27}$ Despite efforts, neither laboratory was able to produce stable isolates with elevated MICs to dalbavancin, suggesting the development of resistance through spontaneous mutation is predicted to be uncommon. Organisms, such as Enterococcus spp. that harbor the vanA gene, are predicted to have elevated MICs to dalbavancin because the direct activity of vanA results in a modified D-alanyl-D-alanine in the bacteria, thereby reducing the activity of dalbavancin.

\section{Clinical trials in humans}

One of the initial Phase III randomized, noninferiority studies for complicated skin and skin structure infections (cSSSI) was undertaken by Jauregui et $\mathrm{al}^{28}$ in January 2003. This was a multinational, multicenter, double-blinded investigation comparing the dalbavancin two-dose regimen against a 14-day course of linezolid (Table 2). The control group was initiated on intravenous linezolid but transition to oral therapy ("step-down" therapy) was allowed. To maintain blinding, patients in the dalbavancin group were given saline infusions every 12 hours, and when clinically stable stepped down to an oral placebo, whereas the linezolid group received intravenous saline doses on days 1 and 8 . The use of aztreonam or metronidazole was allowed at the investigators discretion if Gram-negative or anaerobic pathogens were suspected to be involved in the cSSSI, but the use of some other agents was prohibited. ${ }^{28}$ For inclusion into the study, patients must have had a diagnosed cSSSI that warranted intravenous

Table 2 Summary Phase III DLB trials

\begin{tabular}{|c|c|c|c|c|c|}
\hline Study (year) & Study design/phase & Treatment groups & Results & Difference $(95 \% \mathrm{Cl})$ & References \\
\hline Jauregui et al (2005) & $\begin{array}{l}\text { Randomized, double-blind, } \\
\text { multicenter, noninferiority } \\
\text { Phase III }\end{array}$ & $\begin{array}{l}\text { DLB I,000 mg day I, then } 500 \mathrm{mg} \text { day } 8 \\
\text { LZD } 600 \mathrm{mg} \mathrm{q} / 2 \mathrm{~h} \times 14 \text { days }\end{array}$ & $\begin{array}{l}I^{\circ} \mathrm{CE}: \\
\text { DLB 88.9\% } \\
\text { LZD } 91.2 \% \\
\text { I }^{\circ} \mathrm{ME}: \\
\text { DLB } 89.5 \% \\
\text { LZD } 87.5 \% \\
\text { Overall: } \\
\text { DLB 88.4\% } \\
\text { LZD } 86.8 \%\end{array}$ & $\begin{array}{l}-2.3(-7.3, N R) \\
-2.0(N R) \\
-0.9(N R)\end{array}$ & 28 \\
\hline DISCOVER I & $\begin{array}{l}\text { Randomized, double-blind, } \\
\text { multicenter, noninferiority } \\
\text { Phase III }\end{array}$ & $\begin{array}{l}\text { DLB I,000 mg day I, then } 500 \mathrm{mg} \text { day } 8 \\
\text { VAN } \pm \text { LZD } \times 10-14 \text { days }\end{array}$ & $\begin{array}{l}I^{\circ}: \\
\text { DLB 83.3\% } \\
\text { VAN/LZD 8I.8\% } \\
2^{\circ} \text { CE: } \\
\text { DLB 87.0\% } \\
\text { VAN/LNZ 91.4\% } \\
2^{\circ} \text { Investigator: } \\
\text { DLB } 94.7 \% \\
\text { VAN/LNZ 97.5\% }\end{array}$ & $\begin{array}{l}1.5(-4.6,7.9) \\
-4.4(-9.6,1.6) \\
-2.8(-6.7,0.7)\end{array}$ & 29 \\
\hline DISCOVER 2 & $\begin{array}{l}\text { Randomized, double-blind, } \\
\text { multicenter, noninferiority } \\
\text { Phase III }\end{array}$ & $\begin{array}{l}\text { DLB I,000 mg day I, then } 500 \mathrm{mg} \text { day } 8 \\
\text { VAN } \pm \text { LZD } \times 10-14 \text { days }\end{array}$ & $\begin{array}{l}I^{\circ}: \\
\text { DLB 79.8\% } \\
\text { VAN/LNZ 78.3\% } \\
2^{\circ} \text { CE: } \\
\text { DLB 93.5\% } \\
\text { VAN/LNZ 92.7\% } \\
2^{\circ} \text { Investigator: } \\
\text { DLB } 96.9 \% \\
\text { VAN/LNZ 96.0\% }\end{array}$ & $\begin{array}{l}-1.5(-7.4,4.6) \\
0.8(-3.3,4.9) \\
-0.9(-2.2,4.1)\end{array}$ & 29 \\
\hline Dunne et al (2016) & $\begin{array}{l}\text { Randomized, double-blind, } \\
\text { multicenter, noninferiority } \\
\text { Phase III }\end{array}$ & $\begin{array}{l}\text { DLB I,000 } \mathrm{mg} \text { day I, then } 500 \mathrm{mg} \text { day } 8 \\
\text { DLB I,500 } \mathrm{mg} \times \mathrm{I} \text { dose }\end{array}$ & $\begin{array}{l}I^{\circ}: \\
81.4 \% \text { DLB I dose } \\
84.2 \% \text { DLB } 2 \text { dose } \\
2^{\circ} \text { Success I } 4 \text { day: } \\
84.1 \% \text { DLB I dose } \\
84.8 \% \text { DLB } 2 \text { dose } \\
2^{\circ} \text { Success } 28 \text { day: } \\
84.5 \% \text { DLB I dose } \\
85.1 \% \text { DLB } 2 \text { dose }\end{array}$ & $\begin{array}{l}-2.9(-8.0,2.8) \\
-0.9(-6.3,4.6) \\
-0.6(-6.0,4.8)\end{array}$ & 30 \\
\hline
\end{tabular}

Abbreviations: $I^{\circ}$, primary outcome; $2^{\circ}$, secondary outcome; CE, clinically evaluable; DLB, dalbavancin; LZD, linezolid; ME, microbiologically evaluable; NR, not reported; VAN, vancomycin; Cl, confidence interval. 
therapy targeted against Gram-positive pathogens. Only patients with complicated cSSSI as defined in the protocol were eligible for enrollment. The protocol required that the cSSSI involved deeper layers of the soft tissue or infections requiring surgical interventions. In addition to the protocol cSSSI, patients must have had at least one predefined systemic presentation as well as at least two local signs of infection such as tenderness, drainage, or heat/warmth. Patients with more complicated infections requiring multiple surgical interventions, osteomyelitis, or septic joint infections were excluded from participating in the study. The primary outcome was clinical success during the test of cure visit, which was required between days 14 and 16 after completion of treatment. Secondary endpoints included microbiological cure as well as the drug safety and tolerability. ${ }^{28}$ Of the initially enrolled 854 patients, 77\% (660/854) were eligible for inclusion in the primary endpoint. About $10 \%$ of patients were excluded due to administration of prohibited antimicrobial agents. Of the included patients for evaluation, baseline characteristics were well matched between groups, primarily with Caucasian males around 47 years of age. Despite randomization, there were significantly more patients enrolled in the dalbavancin arm who had a history of vascular disease $(11 \%$ vs $6 \% ; P<0.05)$ as compared to the linezolid group. ${ }^{28}$ For the primary end point of clinical success at the end of cure visit, dalbavancin two-dose regimen was found to be noninferior to linezolid with $88.9 \%$ of dalbavancintreated patients determined a success as compared to $91.2 \%$ for the linezolid group. This had a lower limit of the $95 \%$ confidence interval of $-7.3 \%$, meeting the criteria for noninferiority ( $>-10 \%$ margin). In the microbiologically evaluable population, similar efficacy was demonstrated between treatment groups ( $89.5 \%$ vs $87.5 \%$, respectively), including patients whose infection was caused by MRSA. Success was continued in both treatment arms through the late follow-up period ( 1 month postcompletion of therapy) with only an additional $0.6 \%$ of patients from either group reporting treatment failure. ${ }^{28}$ As evaluated by Jauregui et al, ${ }^{28}$ the treatment arms reported similar rates of adverse events; $56 \%$ for dalbavancin compared to $61 \%$ for linezolid. Upon further examination, investigators classified a trend toward more adverse events to be possibly or probably related to linezolid (32.2\%) as compared to dalbavancin (25.4\%), although this did not reach statistical significance. Only $8 \%$ of patients overall reported a serious adverse event, with ultimately only three events attributed to the study medication. Less than $4 \%$ of patients in either treatment regimen discontinued therapy due to an adverse event. Despite the difference in medication half-lives, the duration of documented adverse events was similar between the groups. The pivotal indication studies of dalbavancin for the treatment of ABSSSI are the DISCOVER 1 and DISCOVER 2 Phase III trials. ${ }^{29}$ These two trials were designed in collaboration with the FDA to be identical in protocol to ensure that when analysis was performed, the results would be comparable. Patients considered for enrollment were evaluated by the physician to determine if the patients met the inclusion definition for ABSSSI with one or more systemic sign of infection, as well as if treatment with at least three days of intravenous antimicrobials would be needed. If initial criteria were met, the final step was to ensure patients met the requirements for the FDA definition of ABSSSI. Of note, patients who received previous antibiotic therapy within the immediate 14 days were excluded..$^{29}$ Patients were randomized into two treatment groups: dalbavancin 1,000 mg intravenous on day 1, with supplemental $500 \mathrm{mg}$ dose on day 8 or vancomycin every 12 hours with weight-based dosing by an unblinded pharmacist allowed. As patients were improving, step-down to oral linezolid or placebo was allowed to finish the 10-14-day course. Admission to a hospital was not required for inclusion if arrangements were made to ensure the patient could receive the treatment in compliance with the protocol. The primary end point for these studies was 48-72 hours clinical response defined as the patient being afebrile with cessation of erythema spread. In an attempt to minimize investigator treatment alterations, this end point was determined after the end of therapy competition. Other end points evaluated included clinical success as determined by the investigator at end of therapy as well as a safety analysis. ${ }^{29}$ DISCOVER 1 and DISCOVER 2 enrolled a total of 1,303 patients with 652 randomized to the dalbavancin treatment arm and 651 in the vancomycin/linezolid group. Baseline characteristics both between groups and studies were well matched, although significantly more patients with diabetes mellitus were included in the DISCOVER 2 trial. The most common systemic symptom qualifying for enrollment was fever at baseline $(85 \%)$ followed by infected areas well beyond the required $75 \mathrm{~cm}^{2}$. The average lesion size was 351 and $336 \mathrm{~cm}^{2}$ for DISCOVER 1 and 2, respectively. ${ }^{29}$ The primary outcome in the intent-to-treat population of noninferiority at the 48-72-hour evaluation point was met by dalbavancin, with the early clinical response of $79.7 \%$ compared to $79.8 \%$ for vancomycin ( $95 \%$ confidence interval: -4.5 to 4.2$)$. By the end of therapy, $90.7 \%$ of the dalbavancin group and $92.1 \%$ in the vancomycin/linezolid group were successfully treated. When evaluated by the individual investigators, clinical 
success rates increased to $96.0 \%$ and $96.7 \%$, respectively. Secondary analysis did identify significantly longer duration of therapy in the vancomycin/linezolid treatment group as compared to the dalbavancin $\operatorname{arm}(38.0 \%$ vs $31.0 \% ; P=0.008)$. Table 2 also summarizes briefly the DISCOVER 1 and 2 trials' outcomes. ${ }^{29}$ Similar to previous studies, dalbavancin and the standard therapy of vancomycin/linezolid were each well tolerated in the DISCOVER 1 and 2 trials. Only $2.1 \%$ of dalbavancin and $2.0 \%$ of comparator group patients discontinued treatment due to a drug adverse event. The overall rate of any adverse event as reported by the investigator was slightly lower in the dalbavancin group at $32.8 \%$ compared to $37.9 \%$ in the vancomycin/linezolid $\operatorname{arm}(P=0.05)$, although the blinded evaluators only determined $12.3 \%$ vs $13.7 \%$, respectively $(P=0.45)$ as treatment related. The most common medication-related adverse event was nausea, but this was not different between treatment arms. Significantly fewer patients in the dalbavancin group experienced diarrhea $(0.8 \%$ vs $2.5 \% ; P=0.02)$ or pruritis $(0.6 \%$ vs $2.3 \%, P=0.01)$ compared to the vancomycin/linezolid group. Fewer serious adverse events were reported in patients treated with dalbavancin, at $2.6 \%$, than those receiving vancomycin/linezolid, at $4.0 \%(P=0.16)$; few of these were attributed to the investigational drug by the blinded review board. For patients who did experience a treatment-emergent drug event, despite the significant difference in medication half-lives, the duration of the event was not documented to be any longer in the treatment arm. ${ }^{29}$ Dunne et $\mathrm{al}^{30}$ reported the results of another Phase III study of dalbavancin for the treatment of ABSSSI. This was a randomized, double-blind, multicenter trial evaluating the use of dalbavancin at the standard dose compared to a single $1,500 \mathrm{mg}$ dose for the treatment of ABSSSI. Similar to previous Phase III trials, patients were included if they had ABSSSI involving deeper tissue or requiring significant surgical intervention along with at least one systemic sign of infection (fever, elevated white blood cell count, $>10 \%$ bandemia) and two additional clinical signs of infection including purulent drainage, tenderness to palpation, swelling/indurations, or fluctuance/localized warmth. Patients with suspected osteomyelitis, Gram-negative infections, diabetic foot infections, decubitus ulcers burns, or infected devices were excluded from the study. The primary outcome was to compare the response to therapy between the treatment groups at $48-72$ hours as determined by a $\geq 20 \%$ reduction in lesion size. Other outcomes included clinical success at day 14 and 28, along with the investigator assessment at these time points. In total, 698 patients were included for randomization with 349 patients in each arm.
Summary demographic data were included, as were overall baseline characteristics and past medical history. Primarily, patients were obese, male patients with cellulitis with an average lesion size about $293 \mathrm{~cm}^{2}$. At the 48-72-hour evaluation point, 81.4\% (284/349) of single dose vs $84.2 \%$ (294/349) of two-dose dalbavancin patients demonstrated $\geq 20 \%$ decrease in lesion size from baseline, which met the criteria for noninferiority. Secondary end points also demonstrated similar clinical success rates at 14 and 28 days as listed in Table 3. Adverse events were similar between treatment groups with only $1.7 \%$ vs $1.4 \%$ of treatment-related adverse events resulting in discontinuation of the study medication. ${ }^{30}$ The most common event reported in either group was nausea, similar to previous Phase III studies for dalbavancin. This study supports the recent FDA approval of a single 1,500 $\mathrm{mg}$ dose of dalbavancin for the treatment of ABSSSI.

\section{Safety profile}

Dalbavancin has been relatively well tolerated during its investigational trials. The majority of adverse events reported by investigators during the multiple clinical trials were categorized as either mild or moderate in severity, but a large proportion of events were difficult to even attribute to the study drug as the potential cause. , $28,29,31^{\text {The most }}$ common events reported are summarized in Table 3 and include nausea and vomiting as the highest documented. There were no differences in adverse events reported during the investigational trials between dalbavancin or the comparator group, regardless of study. Infusion-related reactions were specifically evaluated due to the chemical similarity of dalbavancin to other glycopeptides, particularly vancomycin. Although dalbavancin was administered over

Table 3 Adverse events observed in $>1 \%$ of subjects in either treatment group for DUR00I-30I and DUR00I-302

\begin{tabular}{lll}
\hline & $\begin{array}{l}\text { Dalbavancin } \\
\mathbf{( N = 6 5 2 )}\end{array}$ & $\begin{array}{l}\text { Comparator } \\
\mathbf{( N = 6 5 I )}\end{array}$ \\
\hline Nausea & $29(4.4 \%)$ & $32(4.9 \%)$ \\
Vomiting & $13(2 \%)$ & $10(1.5 \%)$ \\
Headache & $26(4 \%)$ & $23(3.5 \%)$ \\
Diarrhea & $8(1.2 \%)$ & $19(2.9 \%)$ \\
$\gamma$-glutamyl transferase increased & $13(2 \%)$ & $12(1.8 \%)$ \\
ALT increased & $13(2 \%)$ & $9(1.4 \%)$ \\
AST increased & $11(1.7 \%)$ & $2(0.3 \%)$ \\
Pruritus & $6(0.9 \%)$ & $18(2.8 \%)$ \\
Rash & $11(1.7 \%)$ & $9(1.4 \%)$ \\
Pyrexia & $8(1.2 \%)$ & $11(1.7 \%)$ \\
Dizziness & $8(1.2 \%)$ & $6(0.9 \%)$ \\
\hline
\end{tabular}

Note: Data from FDA Briefing Document Anti-infective Advisory Committee Meeting - NDA 21-883. ${ }^{22}$

Abbreviations: ALT, alanine transaminase; AST, aspartate transaminase. 
a short duration (30 minutes) and through a peripheral line, there were no documented cases of red-man syndrome as specifically evaluated by Jauregui et al. ${ }^{28}$ Interestingly, in the DISCOVER trials, many of the infusion-related reactions were not actually reported during drug infusion, but were attributed to the indwelling catheter. ${ }^{29}$ Importantly for clinical practice, although dalbavancin is active in the body for significant lengths of time, the duration of any adverse event was similar between dalbavancin and the comparator agent. ${ }^{8,29}$ Recently a pooled analysis of all Phase II and Phase III clinical trials evaluated adverse events in $\sim 3,000$ patients. Dunne et $\mathrm{al}^{32}$ demonstrated that patients receiving dalbavancin were associated with lower adverse event rates than those patients receiving comparator agents (44.9\% vs $46.8 \%$, respectively; $P=0.012$ ). The majority of the studies analyzed were evaluation of infections of the skin; however, patients with catheter-related infections were also included. The duration and timing of the onset of adverse events were similar for patients receiving dalbavancin and the comparator agents. The adverse events attributed to dalbavancin $(>2 \%)$ were similar to those reported in Table 3 with nausea, vomiting, constipation, and rash being the most commonly reported. No red-man syndrome was reported in the dalbavancin group during Phase II/III studies. ${ }^{32}$

\section{Dosing and availability}

Currently there are two dosing schemes for dalbavancin, with the second regimen being recently FDA approved. Given that dalbavancin has such an extraordinarily long half-life compared to many antimicrobial agents, the FDA initially approved a two-dose regimen for adults with ABSSSI of 1,000 $\mathrm{mg}$ dose on day 1 , followed by $500 \mathrm{mg}$ on day 8 to complete the treatment. More recently the second regimen of dalbavancin 1,500 $\mathrm{mg}$ as a single dose was also FDA approved for the same indication. Each of these dosing regimens can be given through a peripheral intravenous line over 30 minutes. Since studies suggest there is some increase in drug AUC in patients with moderate renal impairment, current recommendations are to decrease the two-dose regimen to $750 \mathrm{mg}$ on day 1 and $375 \mathrm{mg}$ on day 8 , or the single dose regimen to $1,125 \mathrm{mg}$ for patients with a creatinine clearance of less than $30 \mathrm{~mL} / \mathrm{min}$. Patients who are receiving hemodialysis do not require dose adjustments. Adjustments for other patient-specific factors such as age, race, hepatic impairment, or sex are not recommended due to the minimal alterations these factors have on the pharmacokinetic properties of dalbavancin. ${ }^{8,13}$ Dalbavancin is produced as single-use vials of lyophilized powder, which provides $500 \mathrm{mg}$ of dalbavancin when reconstituted. Vials are to be reconstituted with sterile water and gently swirled to dissolve the powder completely. Vigorous shaking is not recommended because the product has a high risk for foaming. Once dissolved, the reconstituted vial results in a concentration of $20 \mathrm{mg} / \mathrm{mL}$ of dalbavancin, which must be further diluted with $5 \%$ dextrose injection prior to use. Dalbavancin can be diluted to between 1 and $5 \mathrm{mg} / \mathrm{mL}$, meaning volumes as small as $200 \mathrm{~mL}$ for the $1,000 \mathrm{mg}$ dose or as large as $1.5 \mathrm{~L}$ for the $1,500 \mathrm{mg}$ dose can be used. This provides for the opportunity to customize the volume infused to the patient on an individual basis. Final product ready for infusion, or reconstituted but not completely diluted product, is stable under refrigeration $\left(2^{\circ} \mathrm{C}-8^{\circ} \mathrm{C} / 36^{\circ} \mathrm{F}-46^{\circ} \mathrm{F}\right)$ or at room temperature $\left(20^{\circ} \mathrm{C}-25^{\circ} \mathrm{C} / 68^{\circ} \mathrm{F}-77^{\circ} \mathrm{F}\right)$ for 48 hours. Dalbavancin cannot be frozen. Because of high risk of precipitation, dalbavancin should not be coadministered with other saline-based medications. Compatibility of dalbavancin with other medications, including those in 5\% dextrose solutions, has not been established and therefore should not be combined. ${ }^{8}$

\section{Patient (and institution) perspectives}

With the introduction of dalbavancin and other long-acting antimicrobial agents into the armamentarium for the treatment of ABSSSI, new opportunities and perspectives to improve patient outcomes, and potential quality of life have been created. One of the most obvious benefits in use of this agent would be a reduction in the necessity for hospital admission for patients with clinically stable ABSSSI while ensuring patient compliance to therapy. The DISCOVERY trials enrolled $25 \%$ of patients who were never admitted to an institution, but because of the drug properties, the patients had similar successes with therapy. ${ }^{29}$ Compliance on the other hand may be difficult for certain patients who are financially limited, resulting in ineffective therapy, and treatment failures potentially requiring hospitalization. The ability to provide effective therapy prior to leaving the institutional control can significantly decrease compliance issues and potentially, readmissions. Specifically from the patient perspective, the benefits of these long-acting agents like dalbavancin can be numerous, starting with admission avoidance. After receiving the antimicrobial, the patient can go home to their comfortable and familiar surroundings, which may prove beneficial for allowing the patient to heal when nothing further is required. For patients who could do with closer follow-up, dalbavancin creates a situation where the patient only needs to visit the physician twice in 2 weeks, providing a combination monitoring/dose administration visit. Either strategy allows for a faster return to normal activities, which may decrease some of the economic burden associated with illness. Finally, not requiring a central 
line to be placed for long-term medication administration will decrease the risk for significant complications from infusion catheters or new infections. From the health care system perspective, keeping the patient out of the hospital or allowing for an earlier release from hospital admission provides opportunities to allocate limited resources to where needed. Additional benefit from this prospective results in less time a patient spends in the health care environment. Many studies have documented longer exposures to health care institutions result in higher possibilities of developing health-care-acquired conditions such as catheter-associated central line infections, thromboembolism events, or decubitis ulcerations, which are being highly scrutinized by hospital regulatory boards. Preventing any hospital-acquired condition is a priority for health care systems not only for regulatory reasons but also as a benefit of providing proper care. Dalbavancin has significant activities against many of the traditional and drug-resistant pathogens involved in ABSSSI. The long half-life and the weekly dosing of dalbavancin provide multiple advantages in many clinical instances, but the safety, effectiveness, and convenience of its use may result in overuse of the drug in patients without attributable risk of MRSA. The drug can limit the ability of antimicrobial stewardship programs de-escalation activities of antimicrobial agents. In cases when the ABSSSI organisms are identified, a narrower spectrum agent is a more appropriate agent, but the weekly dosing of dalbavancin may impede this change due to convenience. This delicate balancing act is the bane of most antimicrobial stewardship programs and needs to be addressed with any antimicrobial agent. Ultimately, as with all antibiotics, judicious selection of the patients to receive the drug should be considered for the optimal use of dalbavancin.

\section{Conclusion}

Dalbavancin is a promising alternative for the treatment of adults with ABSSSI, which can provide advantages over conventional antibiotics. Unlike vancomycin, dalbavancin utilizes its long half-life to allow for either a single- or twodose regimen mitigating the need for invasive central lines. Compared to individual oral agents, dalbavancin has extended spectrum of antimicrobial activity, and thereby can be a preferred treatment in selected clinical cases. Ultimately, the long-acting, safe, and well-tolerated features of dalbavancin provide clinicians the possibility of treating ABSSSI patients in the emergency department or as an outpatient, shortening or even eliminating hospital stays. In turn, the patient may be more likely to be compliant with course of treatment, especially with the one-dose regimen, and experience fewer and less severe adverse effects. With growing concerns of MRSA and other drug-resistant Gram-positive bacteria in ABSSSI, dalbavancin offers unique advantages and establishes a new treatment paradigm that could prove very advantageous for patients and health care systems together.

\section{Disclosure}

KDL received research funding/sponsorship from Merck \& Co., Inc. (formerly Cubist Pharmaceuticals), Allergan (formerly Activis and Forest Pharmaceuticals), The Medicine's Company and Durata Pharmaceuticals. The author reports no other conflicts of interest in this work.

\section{References}

1. Stevens DL, Bisno AL, Chambers HF, et al. Practice guidelines for the diagnosis and management of skin and soft tissue infections: 2014 update by the Infectious Disease Society of America. Clin Infect Dis. 2014; 59(2):147-159.

2. U.S. Food and Drug Administration. Guidance for industry acute bacterial skin and skin structure infections: developing drugs for treatment. 2013. Available from: http://www.fda.gov/downloads/Drugs/ Guidances/ucm071185.pdf. Accessed April 6, 2016.

3. Pallin DJ, Egan DJ, Pelletier AJ, et al. Increased US emergency department visits for skin and soft tissue infections, and changes in antibiotic choices, during the emergence of community-associated methicillin-resistant Staphylococcus aureus. Ann Emerg Med. 2008; 51:291-299.

4. Wilder JR, Wegener DT, David MZ, et al. A national survey of skin infections, care behaviors and MRSA knowledge in the United States. PLoS One. 2014;9(8):e104277.

5. Duong M, Markwell S, Peter J, et al. Randomized, controlled trial of antibiotics in the management of community-acquired skin abscesses in the pediatric patient. Ann Emerg Med. 2010;55:401-407.

6. Howden BP, Davies JK, Johnson PD, et al. Reduced vancomycin susceptibility in Staphylococcus aureus, including vancomycinintermediate and heterogeneous vancomycin-intermediate strains: resistance mechanisms, laboratory detection, and clinical implications. Clin Microbiol Rev. 2010;23(1):99-139.

7. Zhanel GG, Calic D, Schweizer F, et al. New lipoglycopeptides: a comparative review of dalbavancin, oritavancin and telavancin. Drugs. 2010; 70(7):859-886.

8. Dalvance ${ }^{\mathrm{TM}}$ [package insert]. Chicago, IL: Durata Therapeutics. 2014.

9. Bailey J, Summers KM. Dalbavancin: a new lipoglycopeptide antibiotic. Am J Health Syst Pharm. 2008;65:599-610.

10. Malabarba A, Ciabatti R. Glycopeptide derivatives. Curr Med Chem. 2001; 8:1759-1773.

11. Malabarba A, Goldstein BP. Origin, structure and activity in vitro and in vivo of dalbavancin. J Antimicrob Chemother. 2005;55(Suppl 2): ii15-ii20.

12. Chen AY, Zervos MJ, Vazquez JA. Dalbavancin: a novel antimicrobial. Int J Clin Pract. 2007;61(5):853-863.

13. Marbury T, Dowell JA, Seltzer E, et al. Pharmacokinetics of dalbavancin in patients with renal or hepatic impairment. J Clin Pharmacol. 2009; 49(4):465-476.

14. Buckwalter M, Dowell JA. Population pharmacokinetic analysis of dalbavancin, a novel lipoglycopeptide. J Clin Pharmacol. 2005;45: 1279-1287.

15. Dorr MB, Jabes D, Cavaleri M, et al. Human pharmacokinetics and rationale for once-weekly dosing of dalbavancin, a semi-synthetic glycopeptide. J Antimicrob Chemother. 2005;55(Suppl 2):ii25-ii30.

16. Cavaleri M, Riva S, Valagussa A, et al. Pharmacokinetics and excretion of dalbavancin in the rat. J Antimicrob Chemother. 2005;55(Suppl 2): ii31-ii35. 
17. Nicolau D, Sun H, Seltzer E, et al. Pharmacokinetics of dalbavancin in plasma and skin blister fluid. J Antimicrob Chemother. 2007;60: 681-684.

18. Streit JM, Fritsche TR, Sader HS, Jones RN. Worldwide assessment of dalbavancin activity and spectrum against over 6,000 clinical isolates. Diagn Microbiol Infect Dis. 2004;48:137-143.

19. Bowker KE, Noel AR, MacGowan AP. Pharmacodynamics of dalbavancin studied in an in vitro pharmacokinetic system. $J$ Antimicrob Chemother. 2006;58:802-805.

20. Andes D, Craig WA. In vivo pahramcodynamic activity of the glycopeptides dalbavancin. Antimicrob Agents Chemother. 2007;51(5): 1633-1642.

21. Citron DM, Tyrell KL, Goldstein EJ. Comparative in vitro activities of dalbavancin and seven comparator agents against 41 Staphylococcus species cultured from osteomyelitis infections and 18 VISA and hVISA strains. Diagn Microbiol Infect Dis. 2014;79:438-440.

22. FDA Briefing Document Anti-infective Advisory Committee Meeting - NDA 21-883: Dalvance (Dalbavancin) for injection. March 31, 2014. Available from: http://www.fda.gov/downloads/ AdvisoryCommittees/CommitteesMeetingMaterials/Drugs/AntiInfectiveDrugsAdvisoryCommittee/UCM390792.pdf. Accessed April 6, 2016.

23. Goldstein EJ, Citron DM, Merriam CV, et al. In vitro activities of dalbavancin and nine comparator agents against anaerobic gram-positive species and Corynebacteria. Antimicrob Agents Chemother. 2003;47(6): 1968-1971.

24. Leuthner KD, Yuen A, Mao Y, Rahbar A. Dalbavancin (BI-387) for the treatment of complicated skin and skin structure infection. Expert Rev Anti Infect Ther. 2015;13(2):149-159.
25. Johnson DM, Fritsche TR, Sader HS, Jones RN. Evaluation of dalbavancin in combination with nine antimicrobial agents to detect enhanced or antagonistic interactions. Int J Antimicrob Agents. 2006;27:557-560.

26. Lopez S, Hackbarth C, Romano G, Trias J, Jabes D, Goldstein BP. In vitro antistaphylococcal activity of dalbavancin a novel glycopeptides. J Antimicrob Chemother. 2005;55(Suppl 2):ii21-ii24.

27. Goldstein BP, Draghi DC, Sheehan DJ, Hogan P, Sahm DF. Bactericidal activity and resistance development profiling of dalbavancin. Antimicrob Agents Chemother. 2007;51(4):1150-1154.

28. Jauregui LE, Babazadeh S, Seltzer E, et al. Randomized, double-blind comparison of once-weekly versus twice-daily linezolid therapy for the treatment of complicated skin and skin structure infections. Clin Infect Dis. 2005;41:1407-1415.

29. Boucher HW, Wilcox M, Talbot GH, Puttagunta S, Das AF, Dunne MW. Once-weekly dalbavancin versus conventional therapy for skin infection. N Engl J Med. 2014;370(23):2169-2179.

30. Dunne MW, Puttagunta S, Giordano P, et al. A randomized clinical trial of single-dose versus weekly dalbavancin for treatment of acute bacterial skin and skin structure infection. Clin Infect Dis. 2016;62(5): 545-551.

31. Seltzer E, Dorr MB, Goldstein BP, et al. Once-weekly dalbavancin versus standard of care antimicrobial regiments for treatment of skin and soft tissue infections. Clin Infect Dis. 2003;37:1298-1303.

32. Dunne MW, Talbot GH, Boucher HW, et al. Safety of Dalbavancin in the treatment of skin and skin structure infections: a pooled analysis of randomized, comparative studies. Drug Saf. 2016;39(2):147-157.
Therapeutics and Clinical Risk Management

\section{Publish your work in this journal}

Therapeutics and Clinical Risk Management is an international, peerreviewed journal of clinical therapeutics and risk management, focusing on concise rapid reporting of clinical studies in all therapeutic areas, outcomes, safety, and programs for the effective, safe, and sustained use of medicines. This journal is indexed on PubMed Central, CAS,

\section{Dovepress}

EMBase, Scopus and the Elsevier Bibliographic databases. The manuscript management system is completely online and includes a very quick and fair peer-review system, which is all easy to use. Visit http://www.dovepress.com/testimonials.php to read real quotes from published authors. 